\title{
Generalized Inverse Reconstruction for Periodic Nonuniform Sampling
}

\author{
J.Y. Luo, L. Lei \\ School of electronics and information engineering \\ Chengdu University \\ Chengdu, China
}

\begin{abstract}
A traditional assumption underlying most data converters is that the signal should be sampled at a rate exceeding twice the highest frequency. In this paper, we employ a method for low-rate sampling of multi- band signals via applying periodic nonuniform sampling in shift-invariant spaces generated by $m$ kernels with period $T$. So, the sampling and reconstruction of signals were transformed into matrix and vector operations, the generalized inverse can be use to find the answer and a interpolator can insure that complete reconstruction will be achieved. Finally, we validate the method in MATLAB; the conclusion of simulation shows the frame-work presented here is feasible.
\end{abstract}

Keywords- generalized inverse reconstruction; periodic nonuniform sampling; shift-invariant spaces; multi- band signals; interpolator

\section{INTRODUCTION}

One goal in designing a software defined radio (SDR) receiver is to move the analog-to-digital converter (ADC) as close as possible to the antenna [1]. With the development of wireless technology, this enables the modulation of narrow-band signals by high carrier frequencies. To demodulate the desired signals, the required sampling rate for the ADC could often be too high to be attained if the Nyquist sampling theorem is to be satisfied [2]. The uniform bandpass sampling method has been proposed to figure out the problem [3] . The uniform bandpass sampling is the intentional aliasing of the information bandwidth of the signal [4]. The sampling frequency requirement is no longer based on the frequency of the RF carrier, but rather on the information bandwidth of the signal. Thus, the resulting processing rate can be significantly reduced. However, the uniform sampling still suffers from many constraints such problem of timing jitter in $A / D$ conversion process [5].

A signal class that plays an important role in sampling theory is signals in shift-invariant (SI) spaces [6]. A sample in shift-invariant spaces was proposed to overcome these problems.

This paper is organized as followed. Section II sets up the sampling model. In Section III, we use generalized inverse to recover sampled signals. In section IV, we analyze the reconstruction error. Finally, section V shows simulation results.

\section{PROPOSED SCHEME}

The architecture of parallel sampling system is shown in figure 1 .

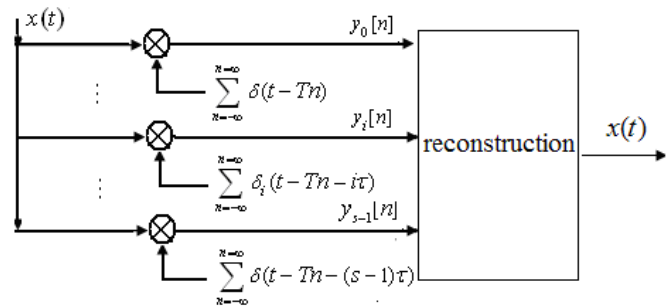

FIGURE I. THE MODE OF THE PERIODIC NONUNIFORM SAMPLING.

The nonuniform sampling process converts a continuous analogue signal $x(t) \in L_{2}$-space into its discrete representation, the architecture of periodic nonuniform sampling system is shown in figure 1.

Let $a_{i}(t)$ as one of $s$ nonuniform sample sequences,

$$
a_{i}(t)=\sum_{n=-\infty}^{+\infty} \delta(t-T n-i \tau)(0 \leq i \leq s-1)
$$

Where, $\mathrm{T}$ is the sampling period, $\tau$ is sequence separation.

One of $s$ sampled functions,

$$
y_{i}(t)=x(n T+i \tau) \sum_{n=-\infty}^{n=\infty} \delta(t-n T-i \tau)
$$

Where, $0 \leq i \leq s-1$.

and the corresponding spectra is given by

$$
Y_{i}(\omega)=\frac{1}{T} \sum_{n=-\infty}^{+\infty} X(\omega-2 \pi n / T) e^{-j 2 \pi n i \tau / T}
$$

In order to reconstruct $x(t)$ from these samples $y[n]\left(y[n]=\left[y_{0}[n], y_{1}[n], \ldots, y_{s-1}[n]\right]\right)$, it is assumed that $x(t)$ lies in a subspace $V(\varphi)$ of $L_{2}$. In this paper, we define that the $V(\varphi)$ are generated by $m$ space functions $\varphi(t)$

$$
V(\varphi)=\left\{\sum_{p=0}^{m-1} \sum_{n \in z} r_{p}[n] \varphi_{p}(t-n T): r_{p}[n] \in L_{2}\right\}
$$

We can represent any $x(t) \in V(\varphi)$ as follow 


$$
x(t)=\sum_{p=0}^{m-1} \sum_{n \in Z} r_{p}[n] \varphi_{p}(t-n T)
$$

The only restriction on the choice of the function $\operatorname{train}\left\{\varphi_{p}(t)\right\}$ is for guaranteeing a unique stable representation of any signal in $V(\varphi)$ by sequence $\left\{r_{p}[n]\right\}$, so the generators $\varphi(t)$ must form a Riesz basis of $L_{2}$. In other words, there exist two constants $\alpha>0$ and $\beta<\infty$, such that:

$$
\alpha\|\mathrm{r}[n]\|_{2}^{2} \leq\left\|\sum_{p=0}^{m-1} \sum_{n \in Z} r_{p}[n] \varphi_{p}(t-n T)\right\|_{2}^{2} \leq \beta\|\mathrm{r}[n]\|_{2}^{2}
$$

Where, $\|\mathrm{r}[n]\|_{2}^{2}=\sum_{p=0}^{m-1} \sum_{n \in z}\left|r_{p}[n]\right|^{2},\|\bullet\|_{2}$ is $L_{2}$ norm.

The above-mentioned subspace $V(\varphi)$ is a single space, the more interesting aspect we are considering is that $x(t)$ lies in a union of subspaces $\bigcup V_{p}(\varphi)(0 \leq p \leq m-1)$

$$
x(t) \in \bigcup V_{p}(\varphi)
$$

In Fourier domain, (4) can be represented as follow:

$$
X(\omega)=\sum_{p=0}^{m-1} R_{p}(\omega) \psi_{p}(\omega)
$$

Where, $R_{P}(\omega)$ is the discrete-time Fourier transform of $r_{p}[n], \psi_{p}(\omega)$ is the Fourier transform of $\varphi_{p}(t)$.

We can obtain the DTFT of the i-th channel samples $y_{i}[n]$ by (3) and (5):

$$
\begin{aligned}
Y_{i}(\omega) & =\frac{1}{T} \sum_{n=-\infty}^{+\infty} \sum_{p=0}^{m-1} R_{p}(\omega-2 \pi n / T) \psi_{p}(\omega-2 \pi n / T) e^{-j 2 \pi n i \tau / T} \\
& =\frac{1}{T} \sum_{p=0}^{m-1} R_{p}(\omega) \sum_{n=-\infty}^{+\infty} \psi_{p}(\omega-2 \pi n / T) e^{-j 2 \pi n i \tau / T}
\end{aligned}
$$

Where, the fact that the $R_{p}(\omega)$ is $2 \pi$-periodic.

An appropriate matrix represent of (7) is given by:

$$
Y(\omega)=H(\omega) R(\omega)
$$

Where, $\quad Y(\omega)=\left(Y_{0}(\omega), Y_{1}(\omega), \cdots Y_{s-1}(\omega)\right)^{\prime}$

$$
\begin{aligned}
R(\omega) & =\left(R_{0}(\omega), R_{1}(\omega), \cdots R_{m-1}(\omega)\right)^{\prime} \\
H(\omega) & =\left[\begin{array}{cccc}
h_{0,0} & h_{0,1} & \ldots & h_{0, m-1} \\
\vdots & \vdots & \ddots & \vdots \\
h_{s-1,0} & h_{s-1,1} & \ldots & h_{s-1, m-1}
\end{array}\right] \\
h_{i, p}(\omega) & =\frac{1}{T} \sum_{n=-\infty}^{+\infty} \psi_{p}(\omega-2 \pi n / T) e^{-j 2 \pi n i \tau / T}
\end{aligned}
$$

Our aim is to obtain values of $R(\omega)$. The method of reconstruction is to solve equation (6).

\section{RECONSTRUCTION MODE}

The approach in this paper is to recovery sampled signals in two steps. First, we use the generalized inverse $H^{-}(\omega)$ to find $r_{i}[n](0 \leq i \leq m-1)$; second, an interpolator is employed to achieve the complete reconstruction of sampled signal..

We define the function as follow:

$$
J=\sum_{i=0}^{s-1} \sum_{n \in Z}\left\|y_{i}[n]-\hat{y}_{i}[n]\right\|^{2}
$$

Where, $\hat{y}_{i}[n]$ is coefficient that is obtained via sampling the reconstructed continuous time signal.

Again by Parseval we have:

$$
\begin{aligned}
J & =\sum_{i=0}^{s-1} \int_{-\pi}^{\pi}\left\|Y_{i}(\omega)-\hat{Y}_{i}(\omega)\right\|^{2} d \omega \\
& =\int_{-\pi}^{\pi}(Y(\omega)-\hat{Y}(\omega))(Y(\omega)-\hat{Y}(\omega))^{H} d \omega
\end{aligned}
$$

Where, $Y_{i}(\omega)$ and $\hat{Y}_{i}(\omega)$ is the DTFT of $y_{i}[n]$ and $\hat{y}_{i}[n]$ respectively. ()$^{\mathrm{H}}$ denotes the Hermitian conjugate.

$$
\begin{gathered}
Y(\omega)=\left(Y_{0}(\omega), Y_{1}(\omega), \cdots, Y_{s-1}(\omega)\right)^{\prime} \\
\hat{Y}(\omega)=\left(\hat{Y}_{0}(\omega), \hat{Y}_{1}(\omega), \cdots, \hat{Y}_{s-1}(\omega)\right)^{\prime} .
\end{gathered}
$$

We have:

$$
\begin{aligned}
\hat{Y}_{i}(\omega) & =\sum_{k=0}^{m-1} \sum_{j=0}^{s-1} Y_{j}(\omega) H_{p, j}^{-}(\omega) \\
& \bullet \sum_{n \in Z} \psi_{p}(\omega-2 \pi n / T) e^{-j 2 \pi n i \tau / T}
\end{aligned}
$$

Where, $H_{p, j}^{-}(\omega)$ is the $p j$ th element of matrix $H^{-}(\omega)$.

A matrix represent of (8) is given by:

$$
\hat{Y}(\omega)=Q(\omega) H^{-}(\omega) Y(\omega)
$$

Where, $Q_{i, p}(\omega)=\sum_{n=-\infty}^{+\infty} \psi_{p}(\omega-2 \pi n / T) e^{-j 2 \pi n i \tau / T}$

Substitute (9) into (7), we have:

$$
\begin{aligned}
J & =\int_{-\pi}^{\pi}\left(Y(\omega)-Q(\omega) H^{-}(\omega) Y(\omega)\right) \\
& \bullet\left(Y(\omega)-Q(\omega) H^{-}(\omega) Y(\omega)\right)^{H} d \omega
\end{aligned}
$$

When the value of the equation (17) is minimum, the generalized inverse can be attained by

$$
H^{-}(\omega)=Q^{H}(\omega) /\left(Q(\omega) Q^{H}(\omega)\right)
$$

As soon as the $r[n]$ is obtained, we can have the recovered $x(t)$ through an interpolator. The $T_{N}$ is defined as the 
oversampling periodic that satisfy $T_{N}=T / M$, we can rewrite (3) as follow:

$$
x\left[n M T_{N}\right]=\sum_{p=0}^{m-1} \sum_{c \in z} r_{p}[c] \varphi_{p}\left(n T_{N}-c T\right)
$$

Upsampling the sequence $\left(x\left[n T_{N}\right]: n \in Z\right)$ by factor of $M$, the dth sub-sequence is given by

$$
x\left[n M T_{N}+d T_{N}\right]=\sum_{p=0}^{m-1} \sum_{c \in Z} r_{p}[c] \varphi_{p}\left(n M T_{N}+d T_{N}-c T\right)
$$

The DTFT of (10) is

$$
X_{d}(\omega)=\sum_{p=0}^{m-1} R_{p}(\omega) \psi_{p, d}(\omega)
$$

Finally, we can have the reconstructed signals in Fourier domain

$$
\begin{aligned}
x(\omega) & =\sum_{d=0}^{M-1} e^{j \omega d} x_{d}(M \omega) \\
& =\sum_{d=0}^{M-1} e^{j \omega d} \sum_{p=0}^{m-1} R_{p}(M \omega) \psi_{p, d}(M \omega) \\
& =\sum_{p=0}^{m-1} R_{p}(M \omega) \sum_{d=0}^{M-1} e^{j \omega d} \psi_{p, d}(M \omega)
\end{aligned}
$$

\section{SimUlation}

In the section, we will validate the reconstruction algorithm in MATLAB. We design a sampling system that the sampling channels are $s=2$. The corresponding nonuniform sample sequences in Fig.1 are $a_{0}(t)=\delta(t-n T)$ and $a_{1}(t)=\delta(t-n T-\tau)$, we define $\tau=T / 3$ that is the sequence separation between two interleaved uniform sample sequences. The generate functions $\varphi_{0}(t)$ and $\varphi_{1}(t)$ are given as follow:

$$
\begin{aligned}
& \varphi_{0}(t)=\operatorname{sinc}\left(\frac{t}{T}\right) e^{j 2 \pi \frac{2}{3 T} t} \\
& \varphi_{1}(t)=\operatorname{sinc}\left(\frac{t}{T}\right) e^{-j 2 \pi \frac{2}{3 T} t}
\end{aligned}
$$

Where, $T$ is the sampling period.

We suppose that the input multi-band signal

$$
x(t)=\sin \left(\frac{7}{3} \pi \times 10^{8} t\right)+\sin \left(\pi \times 10^{8} t\right)
$$

and the sampling period $T=1 / 10^{8}$. Fig 2 shows the conclusion of simulation. The real lines denote the reconstructed signals and the dashes are the imaginary signals. Fig $2 a$ and Fig $2 b$ show those signals in the first channel and the second channel respectively. Obviously, we can observe that the imaginary signals in Fig 2a and Fig $2 \mathrm{~b}$ are restrained successfully from Fig 2c. By comparing Fig $2 \mathrm{c}$ and Fig 2d, the input multi-band signal $x(t)$ can be recovered completely by using the proposed reconstruction method in this paper.

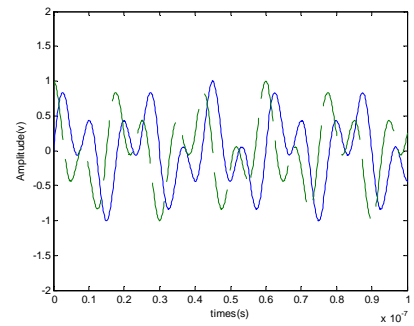

a. First channel signal

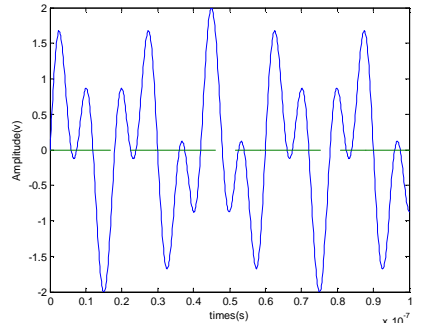

c. Reconstructed signal

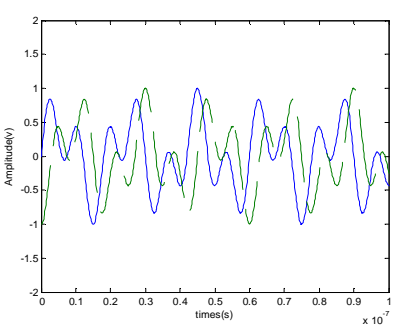

b. Second channel signal

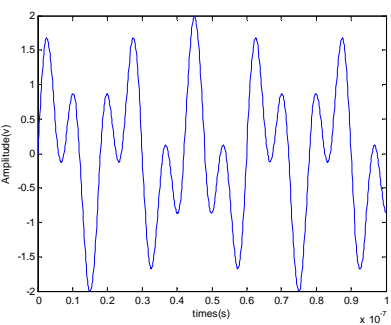

d. Original signal
FIGURE II. THE SAMPLING SYSTEM SIMULATION.

\section{CONCLUSION}

In this paper, we use a general framework to treat sampling of multi-band signal. Our interest is that focused on how to reconstruct signal completely. The latter focuses on using generalized inverse to obtain $r_{i}[n](0 \leq i \leq m-1)$. We showed that by using a interpolator to gain the complete multi-band signal $x(t)$ from $r[n]$. Finally, the simulation proved the method we proposed is feasible.

\section{REFERENCE}

[1] C. H. Tseng and S. C. Chou, "Direct downconversion of multiple RF signals using bandpass sampling," in Proc. ICC, May 2003, vol. 3, pp. 2003-2007.

[2] D. M. Akos, M. Stockmaster, J. B. Y. Tsui, and J. Caschera, "Direct bandpass sampling of multiple distinct RF signals," IEEE Trans. Commun.,

[3] A. J. Coulson, "A generalization of nonuniform bandpass sampling," IEEE Trans. Signal Processing, vol. 43, pp. 694-704, Mar. 1995.

[4] Y.-P. Lin and P. P. Vaidyanathan, "Periodically nonuniform sampling of bandpass signals," IEEE Trans. Circuits Syst. II, vol. 45, pp. 340-351, Mar. 1998.

[5] D. M Bechir,B. R. "Analysis of timing jitter and dither effects on A/D converter for software radio systems" IEEE-ISIVC 2008,Bilbao, Espagne.

[6] W S.Tang. "Oblique projections, biorthogonal Riesz bases andmultiwavelets in Hilbert space". Proceedings of the American Mathematical Society, 2000, 128(2): 463-473. 